\title{
Effect of Bacillus thuringiensis CAB109 on the growth, development, and generation mortality of Spodoptera exigua (Hübner) (Lepidoptera: Noctuidea)
}

\author{
Shichen Huang, Xiangguo Li, Guangchun Li and Dayong Jin*
}

\begin{abstract}
The efficiency of Bacillus thuringiensis (Bt) CAB109 on Spodoptera exigua (Hübner) (Lepidoptera: Noctuidea) larvae was investigated. This study introduces a novel concept of generation mortality (GM) was introduced. Bt CAB109 suspensions at sub-lethal concentrations of $0,10^{2}, 10^{3}, 10^{4}, 10^{5}$, and $10^{6} \mathrm{cfu} / \mathrm{ml}$ were prepared and used to treat the second instar larvae of S. exigua. The results showed that the mortality rates of the larvae were 5.0, 8.3, 15.0, 23.3, 36.7, and $55.0 \%$ respectively after 7 days of treatment. The mean weights of treated larvae with different concentrations were $2.63,2.19,2.03,1.87,1.34$, and $0.96 \mathrm{mg}$ respectively after 6 days, while the developmental durations of such larvae were 16.3, 16.8, 17.5, 18.2, 19.5, and 21.2 days, respectively. Treatment with Bt affected the growth of the larvae at all instars (from the first to the fifth one).

Through the comprehensive interference index of population control (CIIPC), the GM was calculated and the percentages were $30.4,50.3,63.8,77.2$, and $90.6 \%$ for the six tested concentrations respectively. Thus, the GM can be used to evaluate the efficiency of biological pesticides in agricultural practices in the future.
\end{abstract}

Keywords: Bacillus thuringiensis, Spodoptera exigua, Growth development, Generation mortality

\section{Background}

Spodoptera exigua (Hübner) (Lepidoptera: Noctuidea) is a worldwide pest (Da-yong et al. 2009), which mainly attacks vegetables and field crops. Currently, chemical pesticides are being used to control this pest; however, they are not ideal because they cause environmental pollution (Gui-lan et al. 2002). Therefore, it is necessary to use methods that will not pollute the environment (Da-yong and Yong-man 2010 and Da-yong et al. 2012). Bacillus thuringiensis $(B t)$ is currently the most widely used biopesticide (Lan-lan et al. 2008 and Qing-xian 2008). Previous studies showed that $B t$ can not only kill the target pests, but can also inhibit, hinder, and prolong duration of the growth and reproduction of the pests (Barker 1998 and Erb et al. 2001). Therefore, only using the concept of generation mortality to evaluate the effect of chemicals is not sufficient.

\footnotetext{
* Correspondence: jindy@ybu.edu.cn

Yanbian University, Yanbian Korean Autonomous Prefecture, China
}

In the present study, the efficacy of sub-lethal concentrations of $B t \mathrm{CAB} 109$ on the mortality rate, growth, and duration of larvae of S. exigua under laboratory conditions was evaluated.

\section{Materials and methods}

Bacillus thuringiensis (Bt CAB109) was kindly provided by the Laboratory of Pest Biological Control, College of Agriculture and Life Sciences, Chungnam National University (Korea). The Bt CAB109 strain was cultured in Nutrient Agar (NA) medium at $27{ }^{\circ} \mathrm{C}$ for 4 days (when the spore and the parasporal crystal separated from each other) (Da-yong and Yong-man 2010). Next, the culture was washed with sterile water and centrifuged at $4{ }^{\circ} \mathrm{C}$ for $10 \mathrm{~min}$. The supernatant was collected and the concentration of cells in the pellet was about $10^{10} \mathrm{cfu} / \mathrm{ml}$; then the pellet was stored at $4{ }^{\circ} \mathrm{C}$ until further use. S. exigua (larvae and adults) were collected and reared at $25{ }^{\circ} \mathrm{C}$ in $16: 8 \mathrm{~h}$ (light to dark) cycles, and the relative humidity 50 
$60 \%$. The second instar S. exigua larvae were selected for use at different treatments.

\section{Effect on larval growth and development On larval mortality}

The $B t$ suspension was diluted to final concentrations of 0 (control), $1 \times 10^{2}, 1 \times 10^{3}, 1 \times 10^{4}, 1 \times 10^{5}$, and $1 \times 10^{6} \mathrm{cfu} / \mathrm{ml} ; 100 \mu \mathrm{l}$ of the diluted suspension was drawn using a pipette and incubated into artificial feed $(0.5 \mathrm{~g})$ and mixed well. Twenty larvae (second instar) of $S$. exigua that were fasted for $3 \mathrm{~h}$ were placed in a plate with the artificial feed containing concentration of the Bt suspension culture and incubated for $3 \mathrm{~h}$. The larvae were then transferred to a new plate containing artificial feed without $B t$ and reared for 7 days, after which the larval mortality rate was calculated. All the experiments were repeated four times.

\section{On larval weight}

The second instar larvae of S. exigua were divided into groups of 10 and weighed to obtain the average. These groups were treated with $B t$ as described before. Six days later, the groups of treated larvae were weighed and the average of larva weight was estimated. All the experiments were repeated four times.

\section{On larval duration}

Twelve of the second instar S. exigua larvae were treated with $B t$ for $3 \mathrm{~h}$, after which they were transferred into a 12-well culture plate with the diet and reared until pupation, and the larval duration was calculated. All experiments were repeated four times.

\section{Generation mortality (GM)}

GM is the mortality or decrease of the pest numbers at various growth stages (egg, larva, pupa, and adult) in one generation after treating the second instar larvae of S. exigua with Bt CAB109, which was calculated according to the theory of interference index of population control (IIPC) defined by Xiong-fei et al. (2000).

\section{On larvae}

The $B t$ suspension was diluted to final concentrations of 0 (control), $10^{2}, 10^{3}, 10^{4}, 10^{5}$, and $10^{6} \mathrm{cfu} / \mathrm{ml}$; then second instar larvae were treated as mentioned before and kept for 7 days, after which the number of live larvae was calculated; all experiments were repeated three times.

\section{On pupae}

The second instar larvae of S. exigua were treated as described before and reared until pupation. The pupae were then transferred into a new plate and kept until emergence of adults. The ratio of live pupae was then determined. All experiments were repeated three times.

\section{On adults}

The adults were then transferred into a plate and the numbers of live adults in each concentration were calculated. All experiments were repeated three times.

\section{On eggs}

The normal adults were grouped in 1:1 female to male ratio in a plate and provided with absorbent cotton containing $10 \%(w / v)$ glucose solution. The number of eggs laid was estimated. All experiments were repeated three times.

\section{Formula used for the calculations}

$$
\begin{aligned}
& \text { Interference Index of Population Control (IIPC) } \\
& =\frac{\text { survival rate of treated group }}{\text { survival rate of treated group }}
\end{aligned}
$$

Comprehensive interference index of population control (CIIPC)

$$
\begin{aligned}
\mathrm{CIIPC}=\mathrm{IIPC}_{1} \times \mathrm{IIPC}_{2} \times \mathrm{IIPC}_{3} \times \mathrm{IIPC}_{4} \\
\text { where IIPC } \mathrm{II}_{1}=\frac{\text { larvae survival rate of treated group }}{\text { larvae survival rate of control group }} \\
\mathrm{IIPC}_{2}=\frac{\text { pupae survival rate of treated group }}{\text { pupae survival rate of control group }} \\
\mathrm{IIPC}_{3}=\frac{\text { adults survival rate of treated group }}{\text { adults survival rate of control group }} \\
\mathrm{IIPC}_{4}=\frac{\text { eggs number of treated group }}{\text { eggs number of control group }}
\end{aligned}
$$

\section{Generation mortality}

$$
\mathrm{GM}=(1-\mathrm{CIIPC}) \times 100 \%
$$

\section{Data analysis}

The data were analyzed using the OriginPro 9.0 software.

\section{Results and discussion}

\section{Effect on larval mortality}

Data of larval mortality are shown in Fig. 1. A significant difference was found between the results of the treatments when the $B t$ CAB109 concentration was more than $10^{3} \mathrm{cfu} / \mathrm{ml}$. Furthermore, at $10^{3} \mathrm{cfu} / \mathrm{ml}$, the mortality showed an obvious increase.

\section{Effect on larval weight}

The larvae were first treated with $B t$ CAB109 at different concentrations for $2 \mathrm{~h}$ then were reared for 6 days. The survived larvae were weighed. It was found that the average weight of the survived larvae was lower than that of 


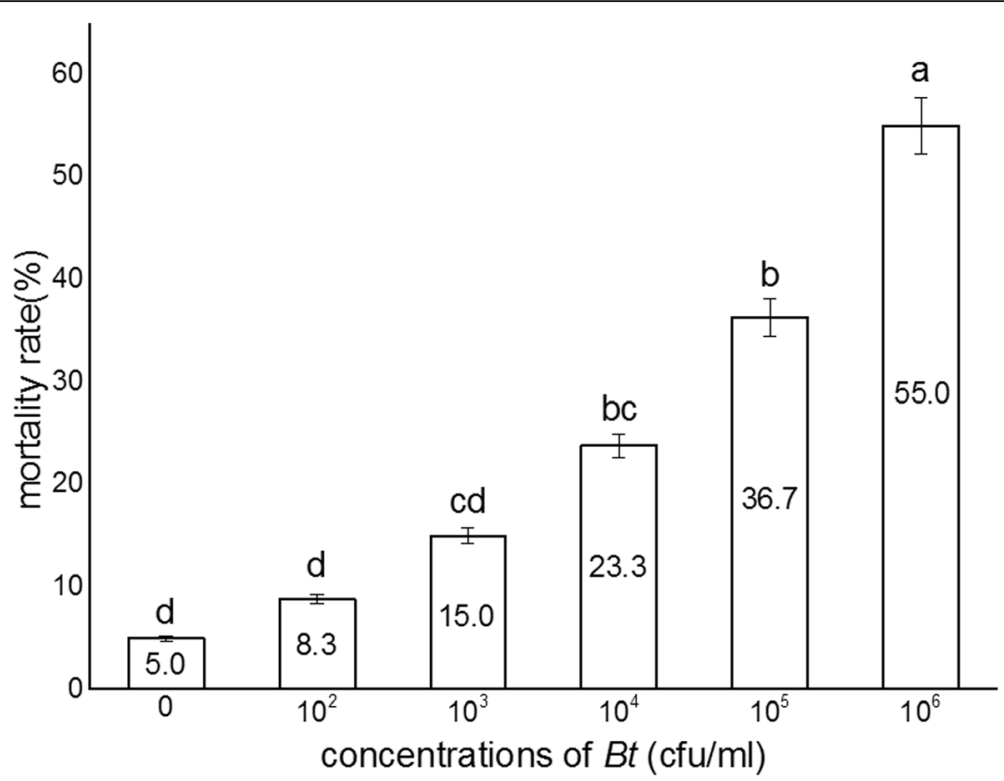

Fig. 1 Effect of Bt CAB109 on the mortality rate of S. exigua larvae

the control and the increase of $B t$ concentration resulted in decrease of the larval weight (Fig. 2).

The weight of each treated larva (in Fig. 2) was calculated using the following equation: $\mathrm{W}=\mathrm{W} 6-\mathrm{W} 0$, where W0 is the weight before treatment, and W6 is the weight of the larvae treated with $B t$ for 6 days.

\section{Effect on larval duration}

The duration of larvae treated with $B t$ increased with increase in concentration. The prolongation of treatment time caused significant differences among treatments (Fig. 3). The durations were 5,3 , and 2 days at $B t$ concentrations of $1 \times 10^{6}, 1 \times 10^{5}$, and $1 \times 10^{4} \mathrm{cfu} / \mathrm{ml}$ respectively.

\section{Effect on the larvae, pupae, adults, and eggs}

The Bt CAB109 could evidently affect all stages of $S$. exigua (Table 1). The CIIPC value decreased with increase in the concentration of Bt. It was found that the smallest IIPC value had the greatest in survival of all the pest stages.

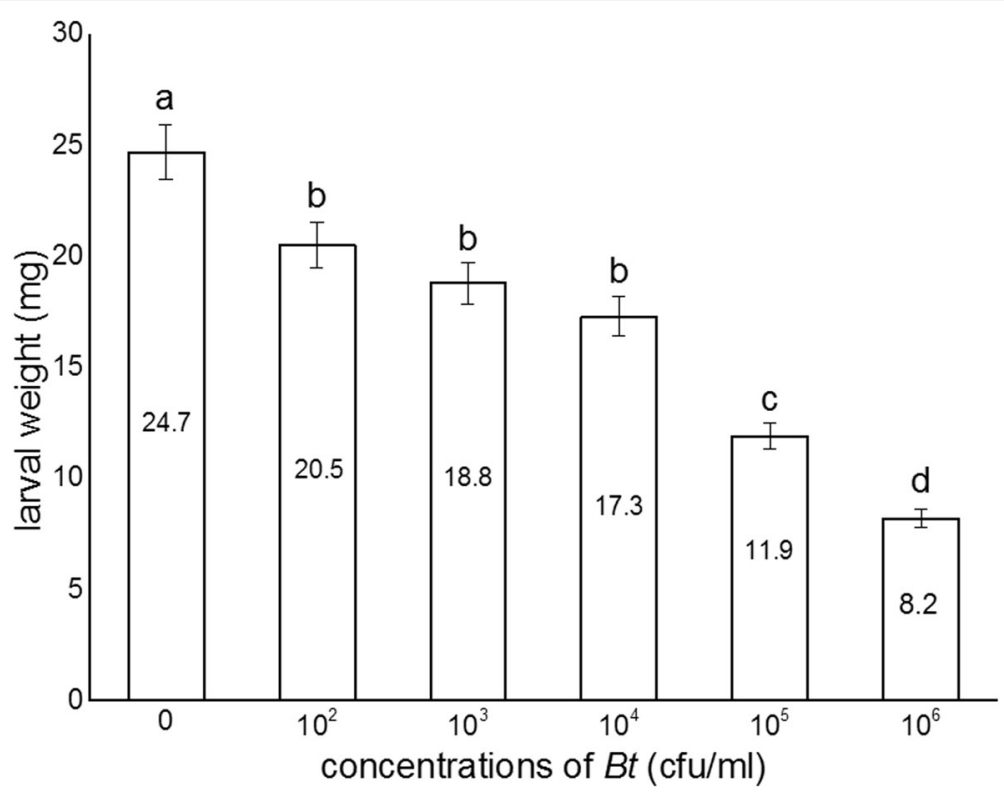

Fig. 2 Effect of Bt CAB109 on body weight of S. exigua larvae 


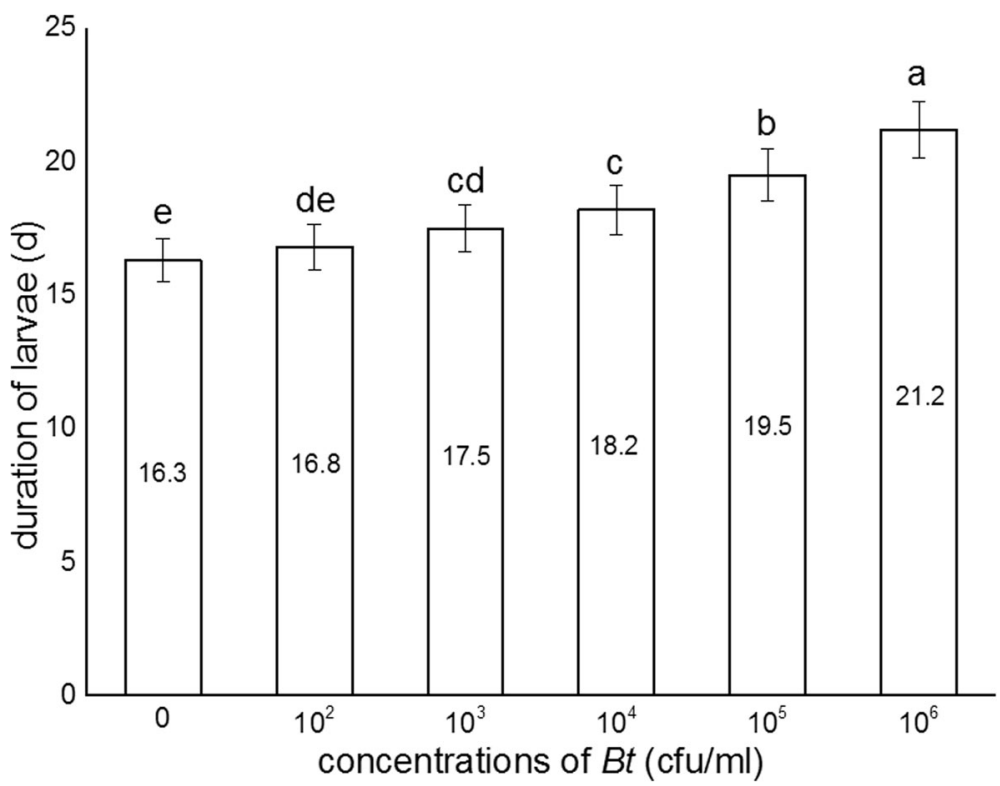

Fig. 3 Effect on Bt CAB109 duration of larvae of S. exigua

\section{Effect on GM}

Bt CAB109 affected not only the larvae, pupae, and adults, but also the eggs laid by the adults (Table 1). Therefore, we introduced the novel concept of GM to explain the relationship between the concentration of $B t$ and stages of $S$. exigua. The GM increased with increase in the concentration of Bt CAB109.

$S$. exigua was reported to have relatively less sensitivity to Bt (Yue-qiu and Xing-fu 2002 and Bao-shan et al. 2006). Our results revealed that unlike insecticides, $B t$ had sublethal effects on $S$. exigua and affected its biological aspects and development. These results are in agreement with those reported by Da-yong and Yongman (2010) who stated that $B t$ affected the growth and development of S. exigua. In addition, Ming et al. (2002) and Donglin et al. (2007) obtained the same results when fed S. exigua larvae on Bt cotton. Although chemical insecticides quickly and efficiently control pests, unlike biological pesticides, the biological pesticides could have a sublethal effect, which could directly affect the weights of the larva, pupa, and the adult pests; growth

Table 1 Effect of different concentrations of Bt CAB109

\begin{tabular}{lllllll}
\hline Con. Bt (cfu/ml) & $\begin{array}{l}\text { Larvae } \\
\mathrm{IPC}_{1}\end{array}$ & $\begin{array}{l}\text { Pupae } \\
\mathrm{IPC}_{2}\end{array}$ & $\begin{array}{l}\text { Adults } \\
\mathrm{IPC}_{3}\end{array}$ & $\begin{array}{l}\text { Eggs } \\
\mathrm{IPC}_{4}\end{array}$ & ClIPC & $\begin{array}{l}\mathrm{GM} \\
(\%)\end{array}$ \\
\hline $1 \times 10^{2}$ & 0.965 & 0.829 & 0.989 & 0.880 & 0.696 & 30.4 \\
$1 \times 10^{3}$ & 0.894 & 0.732 & 0.946 & 0.803 & 0.497 & 50.3 \\
$1 \times 10^{4}$ & 0.807 & 0.659 & 0.896 & 0.759 & 0.362 & 63.8 \\
$1 \times 10^{5}$ & 0.666 & 0.610 & 0.799 & 0.703 & 0.228 & 77.2 \\
$1 \times 10^{6}$ & 0.474 & 0.513 & 0.651 & 0.595 & 0.094 & 90.6 \\
\hline
\end{tabular}

Where $C \mathrm{CIPC}=\| \mathrm{IPC}_{1} \times \mathrm{IIPC}_{2} \times \mathrm{IIPC}_{3} \times \mathrm{IIPC}_{4}$ and $\mathrm{GM}=(1-\mathrm{CIIPC}) \times 100 \%$; con. $B t$ is concentration of $B t$ and development; eclosion rate; egg count; and deformity development, which would inevitably result in the decline in crop yields; moreover, biological pesticides will cause less air pollution than the chemical ones (Shen et al., 1994, Xiao-hui et al. 1999 and Choi et al. 2008). Therefore, it is necessary to evaluate efficient methods for the biological control of pests using pesticides that have the follow-up effect (Shu-liang et al. 2006).

Most target pests would be killed by $B t$, but there are some exceptions such as $S$. exigua, which has relatively lower sensitivity to Bt (Yue-qiu and Xing-fu 2002 and Bao-shan et al. 2006). Moreover, $B t$ was deterrent or feeding inhibition for insects to $B t$. The amount of $B t$ toxin was insufficient to cause the death of insects, but it was enough to affect their normal growth and development (Da-yong and Yong-man 2013). The weight of the insects reduced, which was explained by reduction in food intake. Thus, although the pests were still alive, the degree of harm caused to the plants reduced. At the same time, the development duration of surviving larvae would be prolonged, the generation number would decrease, and the harm to the crops would be reduced.

In this study, with a CAB109 concentration of $1 \times$ $10^{6} \mathrm{cfu} / \mathrm{ml}$, the larval mortality was only $52.6 \%$, but the subsequent actual GM could be up to $90.6 \%$. Therefore, if $B t$ is applied manually, high mortality in a short period cannot be expected. At the time of the occurrence of pests of relatively low density, $B t$ can be proven effective for pest control, with little or no chemical pesticides. Thus, biological pesticides such as $B t$ are important for environmental protection and pollution-free agricultural production (Gay 2012 and Pretali et al. 2016). 


\section{Conclusion}

The generation mortality can be a comprehensive and systematic reflection of the actual control effect of Bacillus thuringiensis for the biological pesticide in the actual control effect, which can make a reasonable assessment.

\section{Acknowledgements}

We thank Professor Youn Young-nam and Dr. Jin Na-young (Chungnam National University, Korea) for their assistance with this study.

\section{Authors' contributions}

SC carried out performed and wrote the paper, XG carried out performed partly, GC participated in the statistical analysis, DY conceived of the study and participated in its design and coordination. All authors read and approved the final manuscript.

\section{Competing interests}

The authors declare that they have no competing interests.

\section{Publisher's Note}

Springer Nature remains neutral with regard to jurisdictional claims in published maps and institutional affiliations.

Received: 26 July 2017 Accepted: 6 December 2017

Published online: 22 February 2018

\section{References}

Bao-shan Y, Lan-juan C, L. (Jun. 2006) Research progress in the control of beet armyworm. J Anhui AgriSci 34(14):3418-3419

Barker J (1998) Effect of Bacillus thuringiensis subsp. kurstaki toxin on the mortality and development of the larval stages of the banded sunflower moth (Lepidoptera: Cochylidae). J Econ Entomol 91:1084-1088

Choi YJ, Gringorten JL, Belanger L, Morel L, Bourque D, Masson L, Groleau D, Miguez CB (2008) Production of an insecticidal crystal protein from Bacillus thuringiensis by the methylotroph Methylobacterium extorquens. Appl Environ Microbiol 74(16):5178-5182

Da-yong J, Seungkyung P, Jinsu K, Suyeon C, Chan P, Taehwan K, Nayoung J, Sunyoung J, Youngnam $Y$, Yongman $Y$ (2009) Environment-friendly control of beet armyworm, Spodoptera exigua (Noctuidae: Lepidoptera) to reduce insecticide use. Appl Entomol 48(2):253-261

Da-yong J, Xueli Q, Xiangguo L, Yongwan Y (2012) Effects of Tween 80 on spreading of Bacillus thuringiensis on crop leaves and its control efficacy against Spodoptera exigua in scallion fields. Plant Prot 38(5):143-146

Da-yong J, Yong-man Y (2010) Isolated and bioassay of Bacillus thuringiensis with high insecticidal activity to Spodoptera exigua. J Agri Sci Yanbian Univ 32(4): 238-242

Da-yong J, Yong-man Y (2013) Effect on growth and development of Spodoptera exigua larvae by Bacillus thuringiensis CAB109. Northern Horticul 20(6):122-124

Donglin H, Jinyu X, Hui L, Wei W, Ji Z, Qian W (2007) Effects of CpTI+Bt transgenie cotton and $B t$ transgenic cotton oil population increase and preference of Spodoptera exigua (Hübner). Acta Phytophy Sin 34(5):461-465

Erb SL, Bourchier RS, Van Frankenhuyzen K, Smith SM (2001) Sublethal effects of Bacillus thuringiensis Berliner subsp. kurstaki on Lymantria dispar (Lepidoptera: Lymantriidae) and the Tachinid parasitoid Compsilura concinnata (Diptera: Tachinidae). Environ Entomol 30(6):1174-1181

Gay H (2012) Before and after silent spring: from chemical pesticides to biological control and integrated pest management-Britain, 1945-1980. Ambix 59(2): 88-108

Gui-lan N, Jian-ping Y, Da-sheng Z, Zhiming Y (2002) Characterization of Bacillus thuringiensis WY-190 showing high performance in killing Spodoptera exigua. Chin J Biol Control 18(4):166-170

Ji-zhong S, Chuan-fan Q, Shu-fang Z (1994) Effects of sub-lethal dosages of Bacillus thuringiensis Galleriae on the metabolism of substances in galleria Mellonella larvae. Acta Phytophylacica Sin. 21(4):373-377

Lan-lan H, Chang-chun D, Fu-ping S, Jie Z, Kui-jun Z (2008) Analysis activity of cry protein from Bacillus thuringiensis against Plutella xylostella of vegetable pest in Heilongjiang Province. Northern Horticul (8):198-200
Pretali LL, Bernardo TS, Butterfield M, Trevisan L, Lucini (2016) Botanical and biological pesticides elicit a similar induced systemic response in tomato (Solanum lycopersicum) secondary metabolism. Phytochemistry 130:56-63

Qing-xian Y (2008) Progress on synergistic bacteria of Bacillus thuringiensis. Northern Horticul (1):55-58

Shu-liang F, Rong-yan W, Jin-yao W, Li-xin D, Da-fang H (2006) Evaluation of control effect of Bacillus thuringiensis strain HBF-1 against larvae of Scarabaeoidae. Acta Phytophylacica Sin 33(4):417-422

Xiao-hui Z, Zi-niu Y, Cui H (1999) Effect of Cry1C toxin from Bacillus thuringiensis on growth, survival and feeding behavior of beet armyworm larva. J Zhejiang Agri Univ 25(1):62-66

Xiong-fei P, Mao-xin Z, You-ming $H$ (2000) Evaluation of plant protectants against pest insects. Chin J Appl Ecol 11(1):108-110

Xue M, Jie D, Cheng-sheng Z (2002) Effect of feeding Bt cotton and other plants on the changes of development and insecticide susceptibilities of lesser armyworm Spodoptera exigua (Hübner). Acta Phytophylacica Sin. 29(1):13-18

Yue-qiu L, Xing-fu J (2002) Biological control of Spodoptera exigua. Plent Protection 28(1):54-56

\section{Submit your manuscript to a SpringerOpen ${ }^{\circ}$ journal and benefit from:}

- Convenient online submission

- Rigorous peer review

- Open access: articles freely available online

- High visibility within the field

- Retaining the copyright to your article

Submit your next manuscript at springeropen.com 